\title{
WDR45 mutations in three male patients with West syndrome
}

\author{
Mitsuko Nakashima ${ }^{1}$, Kyoko Takano ${ }^{2,3}$, Yu Tsuyusaki ${ }^{2}$, Shinsaku Yoshitomi ${ }^{4}$, Masayuki Shimono ${ }^{5}$, \\ Yoshihiro Aoki $^{6}$, Mitsuhiro Kato ${ }^{7,8}$, Noriko Aida ${ }^{9}$, Takeshi Mizuguchi ${ }^{1}$, Satoko Miyatake ${ }^{1}$, Noriko Miyake ${ }^{1}$, \\ Hitoshi Osaka ${ }^{2,10}$, Hirotomo Saitsu ${ }^{1,11}$ and Naomichi Matsumoto ${ }^{1}$
}

West syndrome is an early-onset epileptic encephalopathy characterized by clustered spasms with hypsarrhythmia seen on electroencephalogram (EEG). West syndrome is genetically heterogeneous, and its genetic causes have not been fully elucidated. WD Repeat Domain 45 (WDR45) resides on Xp11.23, and encodes a member of the WD repeat protein interacting with phosphoinositides (WIPI) family, which is crucial in the macroautophagy pathway. De novo mutations in WDR45 cause betapropeller protein-associated neurodegeneration characterized by iron accumulation in the basal ganglia. In this study, we performed whole exome sequencing of individuals with West syndrome and identified three WDR45 mutations in three independent males (patients 1, 2 and 3). Two novel mutations occurred de novo (patients 1 and 2) and the remaining mutation detected in a male patient (patient 3) and his affected sister was inherited from the mother, harboring the somatic mutation. The three male patients showed early-onset intractable seizures, profound intellectual disability and developmental delay. Their brain magnetic resonance imaging scans showed cerebral atrophy. We found no evidence of somatic mosaicism in the three male patients. Our findings indicate that hemizygous WDR45 mutations in males lead to severe epileptic encephalopathy. Journal of Human Genetics (2016) 61, 653-661; doi:10.1038/jhg.2016.27; published online 31 March 2016

\section{INTRODUCTION}

West syndrome is the most common early-onset epileptic encephalopathy with an incidence of $2-5$ per 10000 live births, ${ }^{1,2}$ and is characterized by clustered spasms, hypsarrhythmia patterns on EEG and developmental regression or retardation. ${ }^{3,4}$ As West syndrome is genetically heterogeneous, next generation sequencing has been a powerful tool in detecting causative mutations. ${ }^{5-7}$

WD Repeat Domain 45 (WDR45, MIM: 300526) encodes a WD40repeat protein playing an essential role in the budding of autophagosomes during autophagy. ${ }^{8-13}$ Recent studies have revealed that de novo mutations in WDR45 cause beta-propeller protein-associated neurodegeneration (BPAN), which is a subtype of neurodegeneration with brain iron accumulation (NBIA). ${ }^{14-31}$ This disorder is characterized by early-onset psychomotor retardation followed by progressive motor and cognitive impairment including dystonia, parkinsonism and dementia starting from adolescence or early adulthood. ${ }^{32-34}$ Brain magnetic resonance imaging (MRI) shows distinct T2-weighted signal hypointensity in the globus pallidus and substantia nigra, indicating iron deposition. ${ }^{32-36}$ WDR45 mutations are more common in females, ${ }^{23,31}$ which may be attributed to lethality in males that might be rescued by somatic mosaicism. ${ }^{23,31}$ However, two reported male individuals with hemizygous WDR45 mutations have survived with no evidence of somatic mosaicism. ${ }^{27,28}$

Here, we report three male individuals with West syndrome caused by hemizygous WDR45 mutations exhibiting intractable epilepsy, profound developmental delay and intellectual disability. The existing literature regarding the clinical features of male patients with WDR45 mutations is reviewed and the nature of the mutations, including mosaicism is discussed.

\section{SUBJECTS AND METHODS}

Subjects

A total of 119 individuals with West syndrome were analyzed. Subjects with mutations in known epilepsy-related genes including ARX, KCNT1, KCNQ2, SCN1A, SCN2A, SCN8A, STXBP1, SPTAN1, GNAO1, GRIN1, FOXG1, QARS, EEF1A2, PIGA, CDKL5, SLC35A2, CASK, PCDH19 or MECP2, which were detected by high-resolution melting analysis, target capture sequencing, direct sequencing or whole exome sequencing, were excluded from the study. Clinical

\footnotetext{
${ }^{1}$ Department of Human Genetics, Yokohama City University Graduate School of Medicine, Yokohama, Japan; ${ }^{2}$ Division of Neurology, Kanagawa Children's Medical Center, Yokohama, Japan; ${ }^{3}$ Department of Medical Genetics, Shinshu University School of Medicine, Matsumoto, Japan; ${ }^{4}$ Department of Pediatrics, Shizuoka Institute of Epilepsy and Neurological Disorders, Shizuoka, Japan; ${ }^{5}$ Department of Pediatrics, University of Occupational and Environmental Health, Kitakyushu, Japan; ${ }^{6}$ Department of Pediatrics, Asahi General Hospital, Asahi, Japan; ${ }^{7}$ Department of Pediatrics, Yamagata University Faculty of Medicine, Yamagata, Japan; ${ }^{8}$ Department of Pediatrics, Showa University School of Medicine, Tokyo, Japan; ${ }^{9}$ Department of Radiology, Kanagawa Children's Medical Center, Yokohama, Japan; ${ }^{10}$ Department of Pediatrics, Jichi Medical University, Tochigi, Japan and ${ }^{11}$ Department of Biochemistry, Hamamatsu University School of Medicine, Hamamatsu, Japan

Correspondence: Professor N Matsumoto, Department of Human Genetics, Yokohama City University Graduate School of Medicine, 3-9 Fukuura, Kanazawa-ku, Yokohama 236-0004, Japan.

E-mail: naomat@yokohama-cu.ac.jp

Received 13 January 2016; revised 23 February 2016; accepted 27 February 2016; published online 31 March 2016
} 
information and peripheral blood leukocytes (from all patients and their respective parents), saliva, hair roots and finger nails (only from patient 3 and his mother) were acquired from patients and their family members after receiving their written informed consent. DNA was extracted using QuickGene610L (Fujifilm, Tokyo, Japan) or ISOHAIR (Nippon gene, Tokyo, Japan) according to the manufacturer's instructions. The study was approved by the Institutional Review Board of Yokohama City University School of Medicine and Yamagata University Faculty of Medicine.

\section{Whole exome sequencing}

Patient DNA was captured with the SureSelect Human All Exon V5 kit (Agilent Technologies, Santa Clara, CA, USA) and sequenced on an Illumina HiSeq2500 (Illumina, San Diego, CA, USA) with 101-bp paired-end reads. Exome data processing, variant calling and variant annotation were performed as described previously. ${ }^{24}$ Segregation of WDR45 mutations was examined by Sanger sequencing on an ABI $3130 \times 1$ Genetic Analyzer (Applied Biosystems, Foster City, CA, USA). In two families with a de novo mutation, parentage was confirmed by analyzing 12 microsatellite markers.

\section{Reverse transcription-PCR}

Total RNA was extracted from peripheral blood leukocytes using ISOGEN-LS (Nippon gene) according to the manufacturer's protocol. About $2 \mu \mathrm{g}$ of total RNA was reverse transcribed using the PrimeScript RT reagent kit (TaKaRa Bio, Ohtsu, Japan). The WDR45 gene has two transcript variants, variant 1 (NM_007075.3) has a 1086-bp coding sequence (CDS) over 12 exons (Figure 1d, upper) and variant 2 (NM_001029896.1) has an alternate $5^{\prime}$ untranslated region and 1083-bp CDS over 11 exons lacking 3 bases in the $5^{\prime}$ end of exon 5 compared with the corresponding exon 6 of variant 1 (Figure 1d, lower). WDR 45 transcript variant 2 produces a protein lacking the Ala79 residue that is present in variant 1 . Functional differences between these variants are unknown. Hereafter, the WDR45 mutations described refer to the transcript variant 1 (Figure 1d, upper). We designed target-specific primers to amplify complementary DNA (cDNA) as follows: forward primer spanning exons 2 and 3; 5'-CATTTTATATATATAGGAACAATCCTGC-3' and reverse primer spanning exon 6 and 7; 5'-CGATCACGATCTTGTCATGG-3' (Figure 1d, upper). Two microliters of cDNA was used to amplify the target region and the products were separated by electrophoresis on a $10 \%$ polyacrylamide gel. The products were extracted from gels and cloned into pCR2.1-TOPO TA vectors using the TOPO TA cloning kit (Thermo Fisher Scientific, Waltham, MA), then transfected into Competent Quick DH5 $\alpha$ cells (TOYOBO, Osaka, Japan). Competent cells were spread on Luria-Bertani plates containing ampicillin and incubated at $37^{\circ} \mathrm{C}$ overnight. We performed colony PCRs on randomly selected well-isolated colonies. Plasmid DNA was sequenced using the Sanger method.

\section{Deep sequencing of WDR45 mutations}

Allele frequency of somatic WDR45 mutations was examined by PCR-based deep sequencing using specific primers. Sequencing libraries were prepared using the Nextera DNA Sample Preparation kit (Illumina) and sequenced on a MiSeq (Illumina) with 150 bp paired-end reads. Trimming and alignment of reads were performed as described previously. ${ }^{37}$ Allele counting was performed manually using Integrative Genomics Viewer software (IGV). ${ }^{38,39}$ a

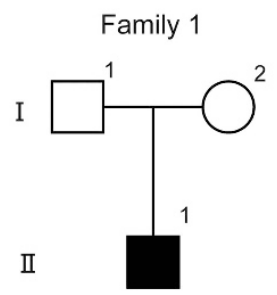

c. $131-1 \mathrm{G}>\mathrm{A}$
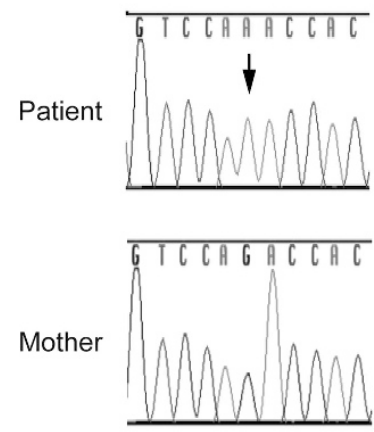

b

Famiy 2

I

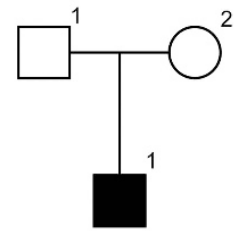

c. $248 \mathrm{G}>\mathrm{A}$
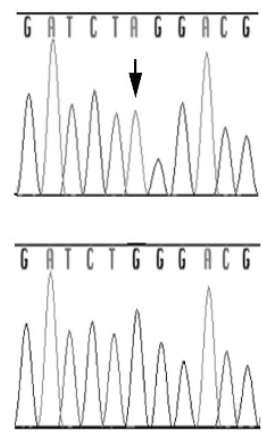

C
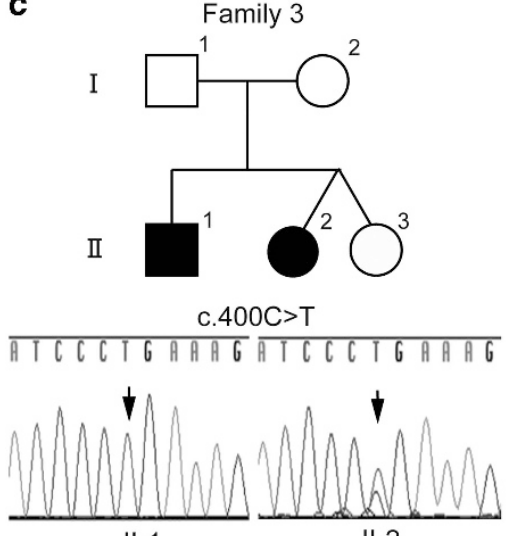

II-1

$\overline{\operatorname{ATCCCCGABAG}}$

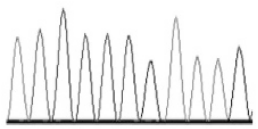

d

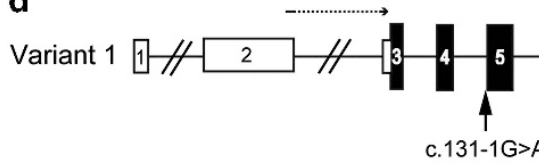

p.

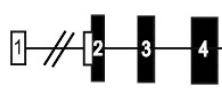

Variant 2

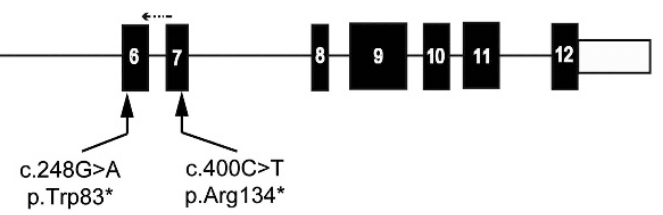

p. $\operatorname{Trp} 83^{*}$

Figure 1 Hemizygous WDR45 mutations in male individuals with West syndrome. Pedigrees (top) and the electropherograms of patients (middle) and their mothers (bottom) of families 1 (a), 2 (b) and 3 (c). (d) Schematic presentation of the WDR45 transcript variant 1 (NM_007075.3, upper) and 2 (NM_001029896.1, lower). The untranslated regions and coding exons are depicted as white and black boxes, respectively. Arrows indicated the mutations described in this study. Dashed arrows indicate the primer set used for RT-PCR analysis. RT-PCR, reverse transcription-PCR. A full color version of this figure is available at the Journal of Human Genetics journal online. 


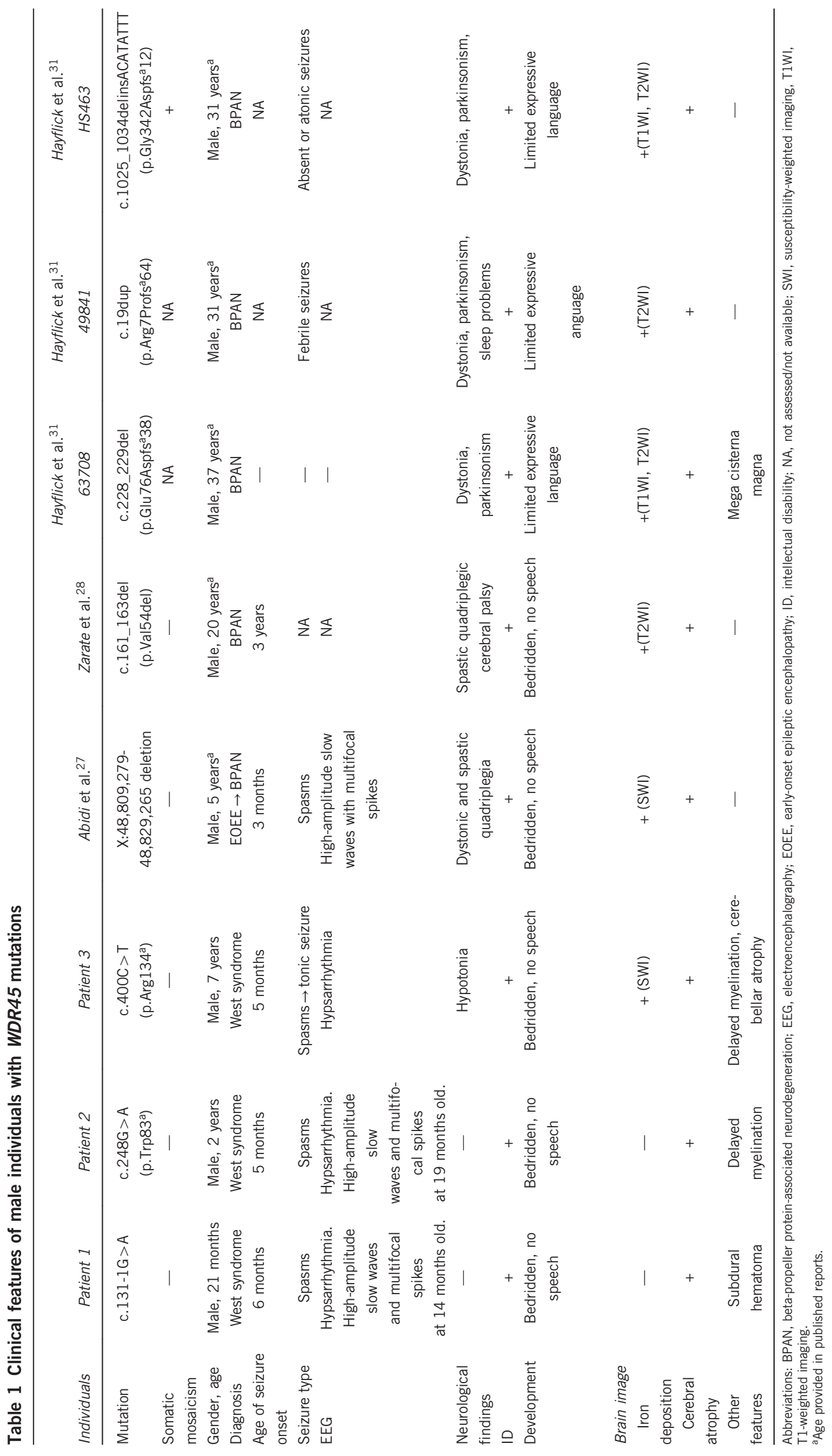




\section{RESULTS}

\section{Case reports}

Clinical features of the three patients described here and five patients previously reported are summarized in Table 1 . All patients had the normal male karyotype (46, XY). Metabolic screening including levels of amino acids, lactic acid, pyruvic acid, ammonia and thyroid function were all normal.

\section{Patient 1}

Patient 1 was a 1-year-old boy (Figure 1a, II-1) and was the first child to non-consanguineous healthy parents. He was born at 39 weeks of gestation without neonatal asphyxia after an uneventful pregnancy. His birth height was $48 \mathrm{~cm}$, weight $3005 \mathrm{~g}$ and head circumference $33 \mathrm{~cm}$. Developmental delay was noticed because of no eye contact and no social smile at 4 months. At 6 months of age, he showed clustered epileptic spasms. Initial EEG revealed hypsarrhythmia, which is characteristic for West syndrome (Figure 2a). His seizures temporarily disappeared with adrenocorticotropic hormone (ACTH) injection for 7 months, but the patient relapsed at 13 months of age. After that, his seizures were refractory to pyridoxal phosphate, valproic acid (VPA) and zonisamide. He showed psychomotor developmental delay with neither visual fixation nor tracking eye movement. Brain MRI at 7 months of age showed delayed myelination in addition to bilateral frontal lobe shrinkage and subdural hematoma around the left frontal lobe associated with ACTH injections (Figure 3a). At 14 months of age, his EEG demonstrated $3-5 \mathrm{~Hz}$ multifocal spike-and-slow-wave bursts on F4-C4, F3-C3, T4-T6 areas with a background of asynchronous left- or right-dominant high-amplitude slow waves $(3-5 \mathrm{~Hz})$. At 16 months of age, his developmental quotient was 12 (Tsumori-Inage scale in Japanese). He was bedridden, not able to rollover, spoke no meaningful words and interacted poorly with others.

\section{Patient 2}

Patient 2 was a 2-year-old boy (Figure 1b, II-1) and was the first child to non-consanguineous healthy parents. He was born at full term without asphyxia after an uneventful pregnancy. His birth height was $49 \mathrm{~cm}$, weight $2598 \mathrm{~g}$ and head circumference $33 \mathrm{~cm}$. At 4 months of age, his development was delayed, with no eye contact, no social smile and no head control. He showed clustered epileptic spasms and hypsarrhythmia on EEG at 5 months of age (Figure 2b). ACTH injection was temporarily effective for both seizures and EEG abnormalities, but his seizures relapsed soon after the injection frequency was reduced. He received a short period of ketogenic diet therapy, which was later discontinued due to a hypoglycemic attack at 7 months of age. His seizures were refractory to treatment from multiple anti-epileptic drugs, including pyridoxine, VPA, clonazepam, nitrazepam and clobazam. His brain MRI revealed delayed myelination at 4 months of age and mild cerebral atrophy at 8 months of age (Figure 3b). At 19 months of age, his EEG demonstrated highamplitude slow waves with multifocal spikes. Currently, he is bedridden without involuntary movement and head control. He was interacted poorly with others and speaks no meaningful words.

\section{Patient 3}

Patient 3 was a 7-year-old boy (Figure 1c, II-1) and was the first child of non-consanguineous parents. His mother had congenital bilateral talipes varus. He was born by spontaneous delivery at 38 weeks without neonatal asphyxia after an uneventful pregnancy. His birth height was $49.5 \mathrm{~cm}$, weight $3172 \mathrm{~g}$ and head circumference $34.5 \mathrm{~cm}$. His facial appearance was normal and he had no external malformations except for an ingrown toenail. Psychomotor retardation was identified by the lack of visual fixation and eye tracking at early postnatal period. At 5 months of age, retinitis pigmentosa-like fundus oculi were noted and seizures with spasms started. At 7 months of age, he experienced clustered epileptic spasms and EEG showed hypsarrythmia (Figure 2c). The electroretinogram indicated poor reaction of rod and cone functions, suggesting Leber congenital amaurosis. The administration of VPA and pyridoxal phosphate had no effect on the symptoms, but ACTH injection temporarily prevented the seizures. After the spasms relapsed at 12 months of age, his seizures were refractory to VPA, zonisamide and lamotrigine. The blood biochemical analysis showed an elevation of serum neuron-specific enolase (NSE, $69.4 \mathrm{ng} \mathrm{ml}^{-1}$, normal range $<15 \mathrm{ng} \mathrm{ml}^{-1}$ ), which is a biomarker for neuroendocrine tumor or brain injury. NSE $\left(50.1 \mathrm{ng} \mathrm{ml}^{-1}\right)$ levels were also elevated in the cerebrospinal fluid, but radiological examination and biochemical screening for neuroblastoma including vanillylmandelic acid and homovanillic acid in the urine were normal. Brain MRI showed delayed myelination and generalized cerebral and cerebellar atrophy at the age of 8 months (Figure 3c). At 4 years and 5 months of age, T2-weighted brain MRI did not detect apparent iron deposition (Figure 3d), but susceptibility-weighted imaging (SWI) implied iron deposition (hypointensity) in the globus pallidus and substantia nigra (Figures $3 e$ and $\mathrm{f}$ ). $\mathrm{He}$ showed global hypotonia, brisk deep tendon reflexes and a Babinski sign was absent. At present, the patient is bedridden and unable to control his head. Profound intellectual disability has been recognized, and he is unable to speak any meaningful words.

His dizygotic twin sister (Figure 1c, II-2) presented with developmental delay and intellectual disability. She was born by cesarean section at 37 weeks of gestation without neonatal asphyxia. Her birth height was $48.5 \mathrm{~cm}$, weight $2404 \mathrm{~g}$ and head circumference $32.5 \mathrm{~cm}$. She had congenital left talipes varus and an ingrown toenail. At 9 and 10 months, and 2 years of age, she had complex febrile convulsions. EEG showed no epileptic activities. At the age of 3 years, her EEG demonstrated frequent focal spike or polyspike and slow wave discharge on P3-O1 and P4-O2 areas. She also showed elevated serum NSE (93.4 $\mathrm{ng} \mathrm{ml}^{-1}$ ), but the brain MRI at 2 years of age was normal. She presented with mild hypotonia and no spasticity. Her developmental milestones were delayed. She could stand at 2 years and 3 months of age. At present, she is unable to speak meaningful words.

\section{Mutation screening}

Whole exome sequencing of 119 individuals with West syndrome identified 3 WDR45 (NM_007075.3) mutations, c.131-1G >A, c. $248 \mathrm{G}>\mathrm{A}\left(\mathrm{p} . \operatorname{Trp} 83^{*}\right)$ and c.400C $>\mathrm{T}\left(\mathrm{p} . \operatorname{Arg} 134^{*}\right)$, in patients 1,2 and 3, respectively (Figure 1d). All mutations were not found in dbSNP137 data, the Exome Aggregation Consortium database (ExAC, Cambridge, MA, USA) or 575 in-house Japanese control exomes. Two mutations, c.131-1G >A and c.248G >A (p.Trp83*), were novel, the other mutation, c.400C $>\mathrm{T}\left(\mathrm{p} . \operatorname{Arg} 134^{*}\right)$, was previously described in two female individuals. ${ }^{18,23,31}$ Sanger sequencing confirmed that the c.131$1 \mathrm{G}>\mathrm{A}$ and c.248G $>$ A mutations occurred de novo. Deep sequencing of PCR products encompassing each mutation amplified using DNA from blood leukocytes in all affected male individuals and saliva, hair root and nail in patients 3 showed that $>99 \%$ of alleles were mutant (Table 2). Approximately $50 \%$ of alleles were mutant in the affected female sibling of patient 3 (Table 2). Consequently, all patients fit to the germline mutations. We were unable to confirm the somatic mosaicism of c.400C $>\mathrm{T}\left(\mathrm{p}\right.$. Arg134 $\left.{ }^{*}\right)$ by Sanger sequencing and high-resolution melting analysis in the healthy mother of patient 3. Deep sequencing was also performed to evaluate the somatic mutation in the mother of patient 3 using different tissues, revealing a low-prevalence somatic mutation in the nails $(0.5-1.0 \%$ of mutant alleles) (Table 2$)$. 
a

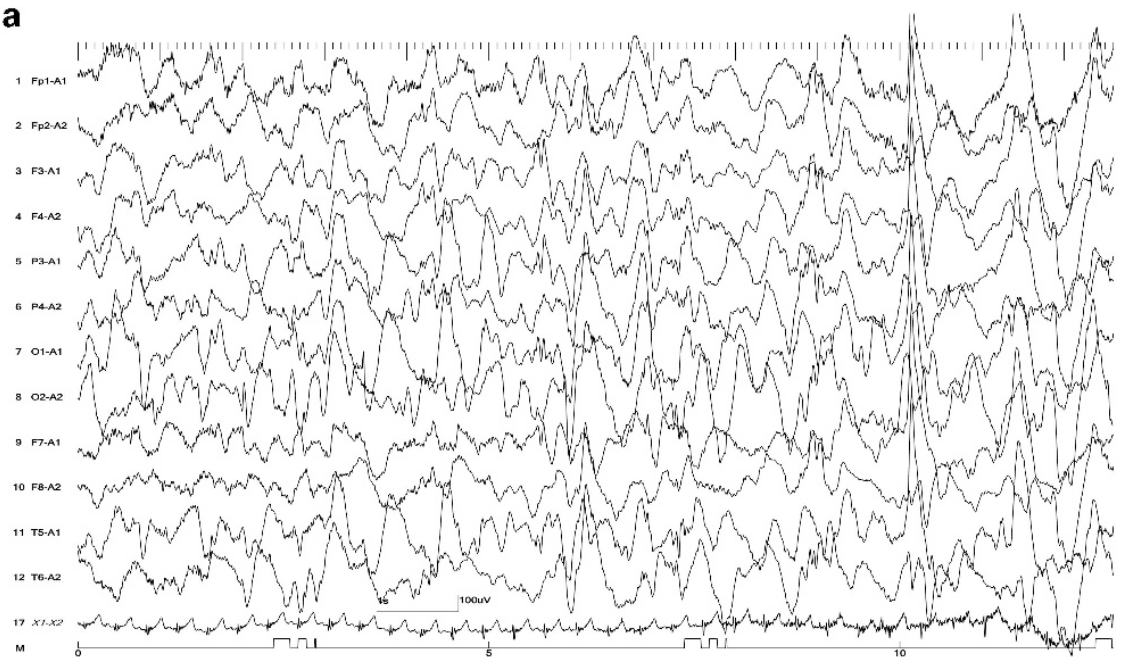

b

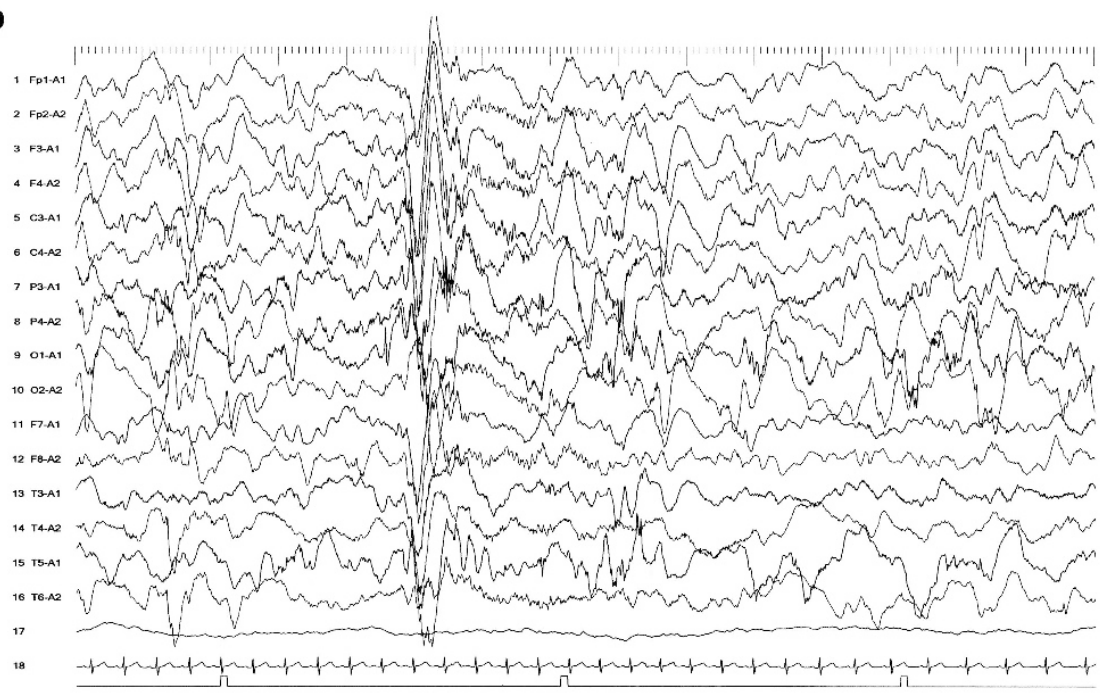

C

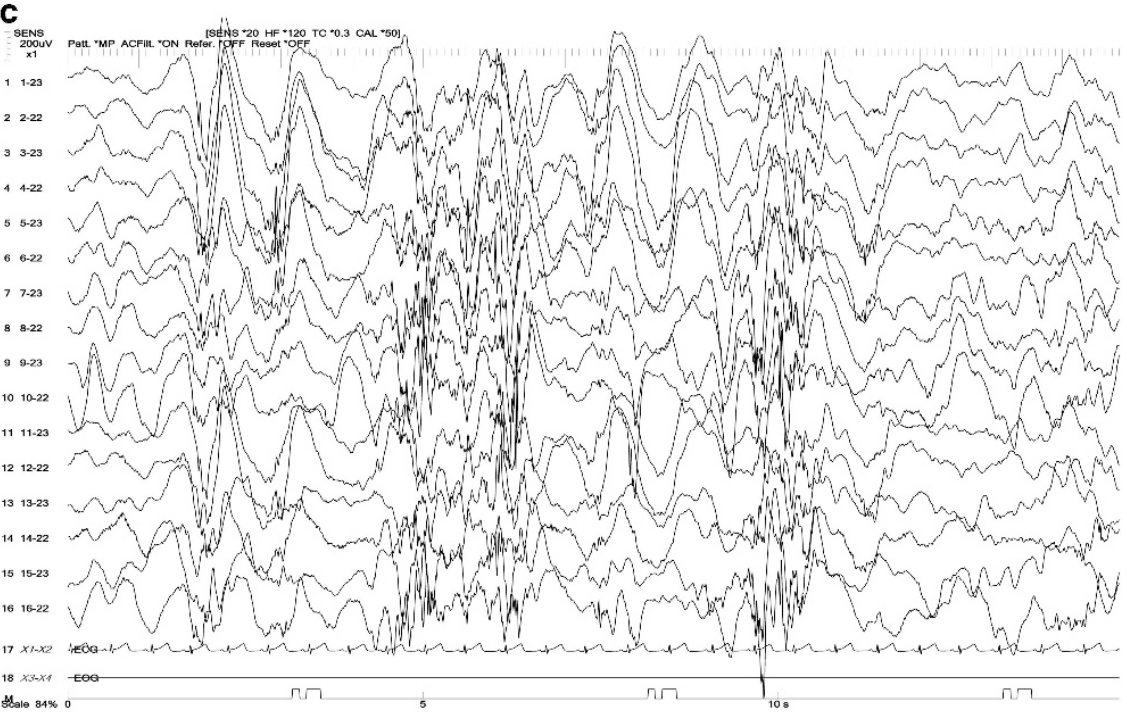

Figure 2 Electroencephalogram (EEG) in males. EEG at 6 months of age in patient 1 (a), 5 months of age in patient 2 (b) and 7 months of age in patient 3 (c). Hypsarrhythmia consisting of high-amplitude irregular slow waves mixed with multifocal spikes or polyspikes were observed in all. 


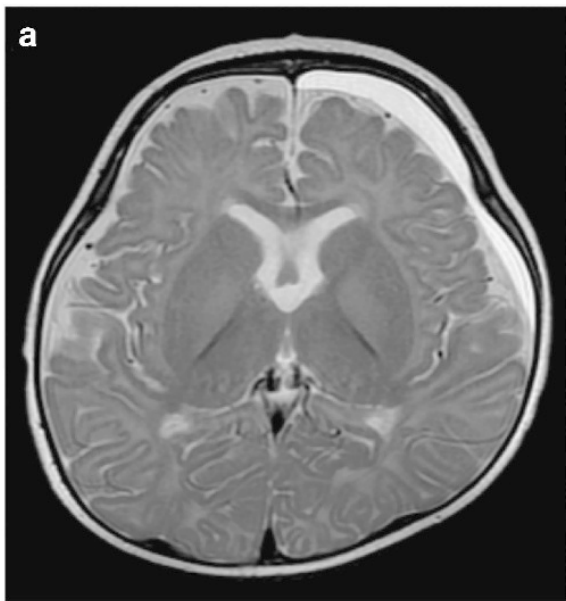

7 months

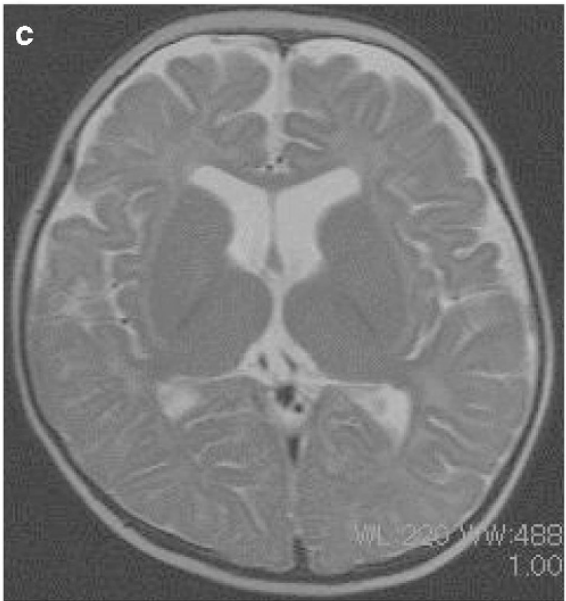

8 months

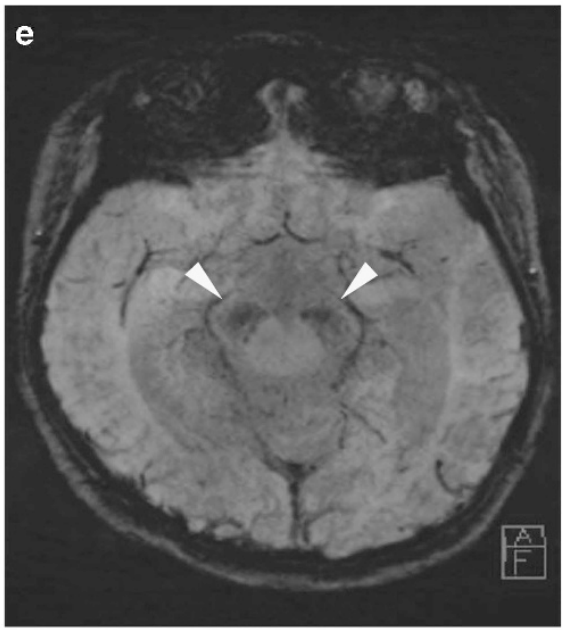

4 years 5 months

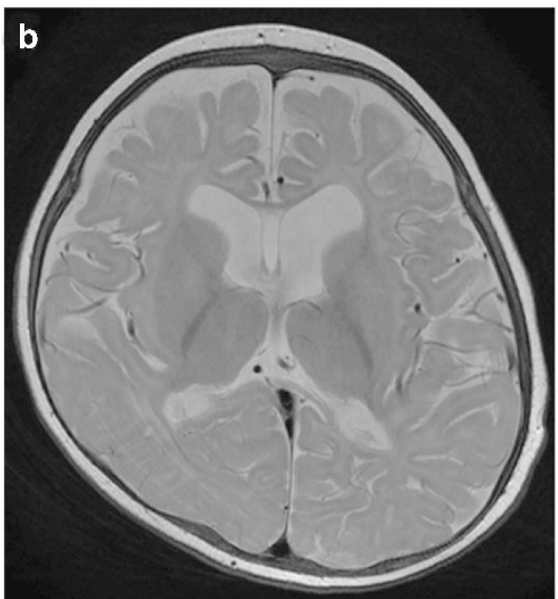

8 months

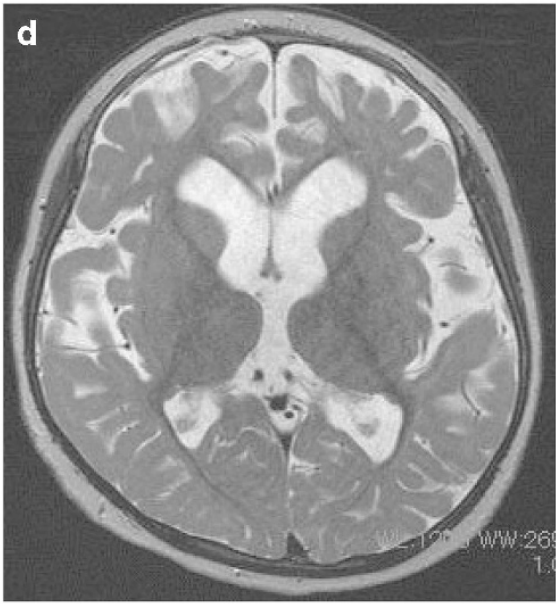

4 years 5 months

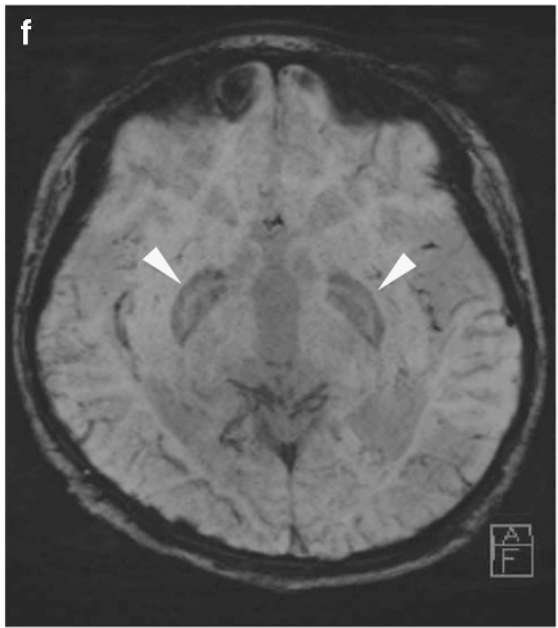

(SWI) brain MRIs (e and $\mathbf{f}$ ) of patient 1 at the age of 7 months (a), patient 2 at the age of 8 months (b), patient 3 at the age of 8 months (c) and the age of 4 years 5 months (d-f). T2-weighted axial view shows delayed myelination with a significant volume loss of deep white matter and mildly enlarged lateral ventricles (a-d). A left frontal subdural hematomais noted in patient 1 (a). In patient 3, SWI showed hypointensity (arrowheads) in the substantia nigra (e) and globus pallidus (f). MRI, magnetic resonance imaging. 


\begin{tabular}{|c|c|c|c|c|c|c|c|c|c|}
\hline \multirow[b]{2}{*}{ Family } & \multirow[b]{2}{*}{ Mutation } & \multirow[b]{2}{*}{ Individual } & \multirow[b]{2}{*}{ Disease status } & \multirow[b]{2}{*}{ Tissue } & \multirow[b]{2}{*}{ Total read depth } & \multicolumn{2}{|c|}{ Wild-type allele } & \multicolumn{2}{|c|}{ Mutant allele } \\
\hline & & & & & & Depth & Frequency $(\%)^{\circledR}$ & Depth & Frequency $(\%)^{a}$ \\
\hline Family 2 & c. $248 \mathrm{G}>\mathrm{A}$ & Patient 2 & Affected & Blood & 54751 & 29 & 0.053 & 54653 & 99.821 \\
\hline \multirow[t]{7}{*}{ Family 3} & c. $400 \mathrm{C}>\mathrm{T}$ & Father & Unaffected & Blood $^{b}$ & 37 141-707 672 & $37073-705130$ & 99.640-99.817 & 28-2339 & $0.075-0.33$ \\
\hline & & Mother & Unaffected & Blood $^{b}$ & 84 414-827 210 & $84286-826239$ & $99.848-99.882$ & $39-834$ & $0.046-0.101$ \\
\hline & & & & Nail $^{\mathrm{b}}$ & $167705-368502$ & $165775-366305$ & 98.849-99.403 & 1696-2042 & $0.554-1.011$ \\
\hline & & Patient 3 & Affected & Blood $^{\mathrm{b}}$ & 51 340-222 715 & 68-316 & $0.014-0.132$ & 51 218-222 060 & $99.471-99.762$ \\
\hline & & & & Salivab & $44098-18331$ & $54-630$ & $0.122-0.344$ & 43 948-182 333 & $99.471-99.659$ \\
\hline & & & & Hairb & 32 244-255 707 & $44-306$ & $0.0112-0.136$ & 32 159-254 752 & 99.626-99.736 \\
\hline & & & & $\mathrm{Nail}^{\mathrm{b}}$ & $125370-164721$ & $112-505$ & $0.067-0.402$ & $124730-164455$ & 99.489-99.838 \\
\hline
\end{tabular}

${ }^{a}$ Frequency (\%) was calculated by wild-type or mutant allele reads/total reads $\times 100$.

bMultiple analyses were performed in these samples $(n=2-5)$.

\section{Transcript analysis}

Two web-based tools (NetGene2 and ESEfinder 3.0) predicted that the c.131-1G > A mutation may lead to aberrant splicing. RT-PCR was performed using cDNA of blood leukocytes from patient 1 and lymphoblastoid cells of a male control. Two different-sized products were amplified in both patient 1 and a control (Figure 4a). We performed sequencing of cloned PCR products and confirmed four different transcripts. Clone 1 derived from band 1 in a control was consistent with the wild-type sequence of WDR45 (Figure 4b, clone 1) and was never observed in patient 1 . Two mutant clones (Figure $4 \mathrm{~b}$, clones 2 and 3) were specific to patient 1 . Clone 2 from band 2 in patient 1 lacked the entire exon 5 of WDR45 variant 1 mRNA (Figure 4b, clone 2). Clone 3 from band 1 in patient 1 showed an 11-bp deletion downstream from the $5^{\prime}$-end of exon 5 in WDR45 variant 2 mRNA (Figure $4 \mathrm{~b}$, clone 3 ). Clone 4 from band 2 in both patient 1 and a control showed skipping of the entire exon 5 in WDR45 variant 2 mRNA (Figure 4b, clone 4). Several WDR45-like pseudogenes exist and the mRNA sequence of WDR45 gene is highly homologous ( $>95 \%$ ) to genomic sequences located at chromosome 4q31.3. Therefore, we estimated this sequence might be erroneously amplified from one of these homologous lesions. Taken together, the splice site mutation led to two aberrant splicing events (clones 2 and 3 ) in patient 1 .

\section{DISCUSSION}

Three male individuals with West syndrome were identified with hemizygous WDR45 mutations. Clinical features of the three individuals, as well as five male individuals previously described with WDR45 mutations or microdeletions are summarized. Developmental delay and intellectual disability were identified in all patients. In this study, developmental milestone was delayed prior to the onset of seizures. The patients either spoke no words or had severely limited language ability. Five individuals were bedridden and three others achieved broad-based or ataxic gait during childhood. Seven of eight patients had seizures, four with epileptic spasms. Dystonia and parkinsonism were documented in three adult individuals. In all cases, brain imaging showed cerebral atrophy. Iron accumulation was observed in five of seven patients, but two of them were only suggested by SWI. Iron accumulation was not confirmed in two of the patients described here, which may be explained by their young ages. The elevation of NSE in serum and cerebrospinal fluid was observed in patient 3 and his affected sister. The previous females with WDR45 mutations also showed mild elevation of NSE. ${ }^{16,21}$ Such cases commonly show no neuroendocrine tumors, indicating that elevated NSE might be induced by the neuronal damage caused by chronic neurological impairment. Takano et al. ${ }^{16}$ proposed that the screening of NSE in serum and cerebrospinal fluid would be helpful for diagnosing BPAN in early childhood. Our findings support this suggestion.

WDR45 mutations are more commonly reported in females than males (5 males and 47 females). ${ }^{15-31}$ Lethality of hemizygous WDR45 mutations may explain this gender bias. Haack et al. ${ }^{23}$ described a male patient (HS463) having a WDR45 mutation in a somatic mosaic manner (Table 1), suggesting that lethality was rescued by the mosaicism. Abidi and Zarate et al. ${ }^{27,28}$ reported a male individual with a hemizygous WDR45 germline mutation that was viable. Therefore, we carefully evaluated the involvement of somatic mosaicism in our patients, but all males had germline mutations (Table 2), supporting the fact that male patients are viable.

Patient 3 with the c.400C > T (p.Arg134*) mutation had an affected female sibling with the same mutation and two female patients with the same c.400C $>\mathrm{T}$ mutation have been previously reported. ${ }^{18,23,31}$ Three female individuals showed developmental delay and intellectual disability, but their phenotype was milder compared with the male patient described in this study. Zarate et al. ${ }^{28}$ also reported a female sibling with an in-frame deletion in WDR45 (c.161_163del:p.Val54del). They described that the male patient exhibited a more severe phenotype than his affected younger sister. ${ }^{28}$ Milder phenotypes in females may be attributed to somatic mosaicism or X-inactivation. Some studies have shown that only random or unfavorable skewing expressed a mutant allele in the DNA of blood leukocytes or lymphoblastoid cells. ${ }^{24,28}$ However, X-inactivation cannot be studied in the brain tissues of patients.

In the family of patient 3 , the healthy mother showed a lowprevalence somatic mutation in nail tissue. Siblings born to a healthy mother carrying a somatic mutation were also described by Zarate et al. ${ }^{28}$ This indicates that parental somatic mosaicism should be taken into account in seemingly 'de novo' mutations. Recently, highly sensitive technologies like deep sequencing and droplet digital PCR have clearly demonstrated that 'de novo' mutations were actually transmitted from apparently healthy parents with low-prevalence somatic mutations. ${ }^{40-42}$ Therefore, we should pay close attention to 


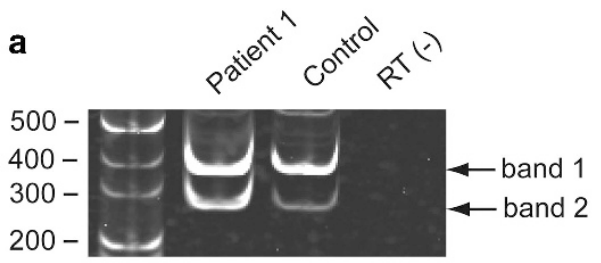

b Clone 1 (Wild-type)
$386 \mathrm{bp}$

Clone 2 (Mutant 1) $281 \mathrm{bp}$

Clone 3 (Mutant 2) $372 \mathrm{bp}$

Clone 4 (Non-specific) 278 bp

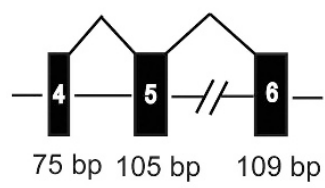

75 bp 105 bp 109 bp

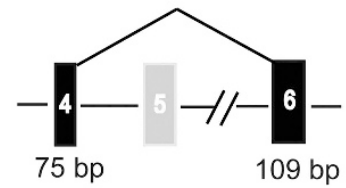

ACCACGAGCAG deletion

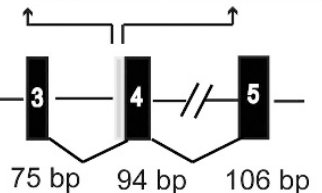

75 bp 94 bp 106 bp
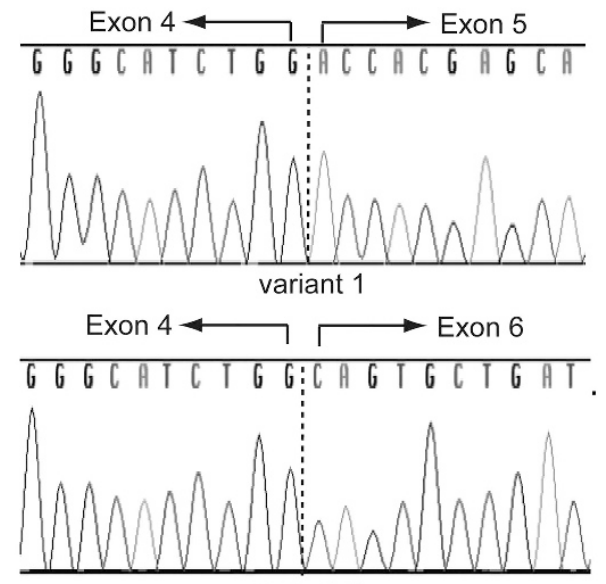

variant 1

Exon $3 \longleftrightarrow$ Exon 4

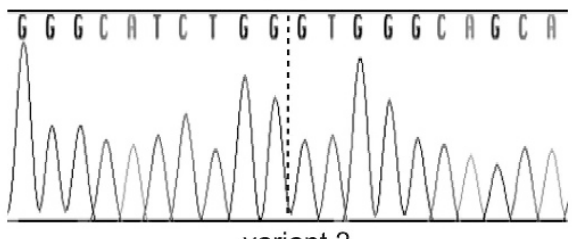

variant 2

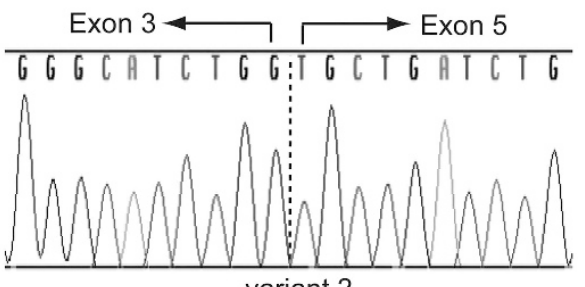

variant 2

Figure 4 Splice donor site mutation (c.131-1G>A) leading to aberrant mRNA splicing in patient 1. (a) RT-PCR analysis using cDNA derived from the patient and a control. Patient and control both showed two bands. (b) Schematic presentation (left) and sequencing results (right) of cloned PCR products. Four different types of clones were found. Clone 1 was derived from band 1 of a control, which is consistent with the wild-type WDR45 transcript variants 1 and 2. Clone 2 obtained from band 2 of patient 1 indicated loss of the entire exon 5 in the WDR45 transcript variant 1 . Clone 3 was obtained from band 1 of patient 1, showing an 11-bp deletion from the $5^{\prime}$-end of exon 5 in WDR45 transcript variant 2. Clone 4 from band 2 of patient 1 and a control showed entire exon 5 skipping in WDR45 transcript variant 2. cDNA, complementary DNA; RT-PCR, reverse transcription-PCR. A full color version of this figure is available at the Journal of Human Genetics journal online.

the recurrence risk in future offspring born to healthy parents with one affected child with a seemingly 'de novo' mutation.

In conclusion, we have identified three male individuals with West syndrome caused by hemizygous germline mutations in the WDR45 gene. WDR45 could be listed as one of the causative genes of West syndrome, and germline mutations in males with more severe features compared with females with the same mutations clearly indicate that males with hemizygous WDR45 mutations can survive.

\section{URLS}

ExAC (http://exac.broadinstitute.org/)

Polyphen-2 (http://genetics.bwh.harvard.edu/pph2/)

MutationTaster (http://www.mutationtaster.org/)
NetGene2 (http://www.cbs.dtu.dk/services/NetGene2/)

ESEfinder 3.0 (http://rulai.cshl.edu/cgi-bin/tools/ESE3/esefinder.cgi? process $=$ home)

\section{CONFLICT OF INTEREST}

The authors declare no conflict of interest.

\section{ACKNOWLEDGEMENTS}

We express our heartfelt gratitude to all the patients and their families for participating in this study. We would like to thank Ms N Watanabe, M Sato and $\mathrm{K}$ Takabe for their excellent technical assistance. We also thank Mr D Yamaguchi for his assistance with the bioinformatics analysis. This work was supported in part by a grant for Research on Measures for Intractable 
Diseases, a grant for Comprehensive Research on Disability Health and Welfare, the Strategic Research Program for Brain Science (SRPBS) and a grant for Initiative on Rare and Undiagnosed Diseases in Pediatrics (IRUD-P) from Japan Agency for Medical Research and Development (AMED); a Grant-in-Aid for Scientific Research on Innovative Areas (Transcription Cycle) from the Ministry of Education, Culture, Sports, Science and Technology of Japan (MEXT); Grants-in-Aid for Scientific Research (A, B and C), and challenging Exploratory Research from the Japan Society for the Promotion of Science (JSPS); the fund for Creation of Innovation Centers for Advanced Interdisciplinary Research Areas Program in the Project for Developing Innovation Systems from the Japan Science and Technology Agency (JST); the Takeda Science Foundation; the Yokohama Foundation for Advancement of Medical Science; and the Hayashi Memorial Foundation for Female Natural Scientists.

1 Saemundsen, E., Ludvigsson, P. \& Rafnsson, V. Risk of autism spectrum disorders after infantile spasms: a population-based study nested in a cohort with seizures in the first year of life. Epilepsia 49, 1865-1870 (2008).

2 Cowan, L. D. \& Hudson, L. S. The epidemiology and natural history of infantile spasms. J. Child Neurol. 6, 355-364 (1991)

3 Osborne, J. P., Lux, A. L., Edwards, S. W., Hancock, E., Johnson, A. L., Kennedy, C. R. et al. The underlying etiology of infantile spasms (West syndrome): information from the United Kingdom Infantile Spasms Study (UKISS) on contemporary causes and their classification. Epilepsia 51, 2168-2174 (2010).

4 Pavone, P., Striano, P., Falsaperla, R., Pavone, L. \& Ruggieri, M. Infantile spasms syndrome, West syndrome and related phenotypes: what we know in 2013. Brain Dev. 36, 739-751 (2014).

5 Epi, K. C., Epilepsy Phenome/Genome P., Allen, A. S., Berkovic, S. F., Cossette, P., Delanty, N. et al. De novo mutations in epileptic encephalopathies. Nature 501 217-221 (2013).

6 Dimassi, S., Labalme, A., Ville, D., Calender, A., Mignot, C., Boutry-Kryza, N. et al. Whole-exome sequencing improves the diagnosis yield in sporadic infantile spasm syndrome. Clin. Genet. 89, 198-204 (2016).

7 Paciorkowski, A. R., Thio, L. L. \& Dobyns, W. B. Genetic and biologic classification of infantile spasms. Pediatr. Neurol. 45, 355-367 (2011).

8 Grimmel, M., Backhaus, C. \& Proikas-Cezanne, T. WIPI-mediated autophagy and longevity. Cells 4, 202-217 (2015).

9 Proikas-Cezanne, T., Takacs, Z., Donnes, P. \& Kohlbacher, O. WIPI proteins: essentia PtdIns3P effectors at the nascent autophagosome. J. Cell Sci. 128, 207-217 (2015).

10 Bakula, D., Takacs, Z. \& Proikas-Cezanne, T. WIPI beta-propellers in autophagy-related diseases and longevity. Biochem. Soc. Trans. 41, 962-967 (2013).

11 Jiang, P. \& Mizushima, N. Autophagy and human diseases. Cell Res. 24 69-79 (2014).

12 Nakatogawa, H., Suzuki, K., Kamada, Y. \& Ohsumi, Y. Dynamics and diversity in autophagy mechanisms: lessons from yeast. Nat. Rev. Mol. Cell Biol. 10 458-467 (2009).

13 Mizushima, N., Levine, B., Cuervo, A. M. \& Klionsky, D. J. Autophagy fights disease through cellular self-digestion. Nature 451, 1069-1075 (2008).

14 Verhoeven, W. M., Egger, J. I., Koolen, D. A., Yntema, H., Olgiati, S., Breedveld, G. J. et al. Beta-propeller protein-associated neurodegeneration (BPAN), a rare form of NBIA novel mutations and neuropsychiatric phenotype in three adult patients. Parkinsonism Relat. Disord. 20, 332-336 (2014).

15 Tschentscher, A., Dekomien, G., Ross, S., Cremer, K., Kukuk, G. M., Epplen, J. T. et al Analysis of the C19orf12 and WDR45 genes in patients with neurodegeneration with brain iron accumulation. J. Neurol. Sci. 349, 105-109 (2015).

16 Takano, K., Shiba, N., Wakui, K., Yamaguchi, T., Aida, N., Inaba, Y. et al. Elevation of neuron specific enolase and brain iron deposition on susceptibility-weighted imaging as diagnostic clues for beta-propeller protein-associated neurodegeneration in early childhood: Additional case report and review of the literature. Am. J. Med. Genet. A 170, 322-328 (2015).

17 Ohba, C., Nabatame, S., Iijima, Y., Nishiyama, K., Tsurusaki, Y., Nakashima, M. et al. De novo WDR45 mutation in a patient showing clinically Rett syndrome with childhood iron deposition in brain. J. Hum. Genet. 59, 292-295 (2014).

18 Nishioka, K., Oyama, G., Yoshino, H., Li, Y., Matsushima, T., Takeuchi, C. et al. High frequency of beta-propeller protein-associated neurodegeneration (BPAN) among patients with intellectual disability and young-onset parkinsonism. Neurobiol. Aging 36, 2004.e9-2004.e15 (2015).

19 Long, M., Abdeen, N., Geraghty, M. T., Hogarth, P., Hayflick, S. \& Venkateswaran, S. Novel WDR45 mutation and pathognomonic BPAN imaging in a young female with mild cognitive delay. Pediatrics 136, e714-e717 (2015).

20 Rathore, G. S., Schaaf, C. P. \& Stocco, A. J. Novel mutation of the WDR45 gene causing beta-propeller protein-associated neurodegeneration. Mov. Disord. 29 574-575 (2014).

21 Okamoto, N., Ikeda, T., Hasegawa, T., Yamamoto, Y., Kawato, K., Komoto, T. et al. Early manifestations of BPAN in a pediatric patient. Am. J. Med. Genet. A 164A 3095-3099 (2014).

22 Khalifa, M. \& Naffaa, L. Exome sequencing reveals a novel WDR45 frameshift mutation and inherited POLR3A heterozygous variants in a female with a complex phenotype and mixed brain MRI findings. Eur. J. Med. Genet. 58, 381-386 (2015).

23 Haack, T. B., Hogarth, P., Kruer, M. C., Gregory, A., Wieland, T., Schwarzmayr, T. et al. Exome sequencing reveals de novo WDR45 mutations causing a phenotypically distinct, X-linked dominant form of NBIA. Am J. Hum. Genet. 91, 1144-1149 (2012).

24 Saitsu, H., Nishimura, T., Muramatsu, K., Kodera, H., Kumada, S., Sugai, K. et al. De novo mutations in the autophagy gene WDR45 cause static encephalopathy of childhood with neurodegeneration in adulthood. Nat. Genet. 45, 445-449, 449e441 (2013).

25 Ryu, S. W., Kim, J. S. \& Lee, S. H. Beta-propeller-protein-associated neurodegeneration: a case of mutation in WDR45. J. Clin. Neurol. 11, 289-291 (2015).

26 Aminkeng, F. WDR45 mutations define a novel disease entity-static encephalopathy of childhood with neurodegeneration in adulthood. Clin. Genet. 84, 209 (2013).

27 Abidi, A., Mignon-Ravix, C., Cacciagli, P., Girard, N., Milh, M. \& Villard, L. Early-onset epileptic encephalopathy as the initial clinical presentation of WDR45 deletion in a male patient. Eur. J. Hum. Genet. 24, 615-618 (2016).

28 Zarate, Y. A., Jones, J. R., Jones, M. A., Millan, F., Juusola, J., Vertino-Bell, A. et al. Lessons from a pair of siblings with BPAN. Eur. J. Hum. Genet. 24 1080-1083 (2016).

29 Ozawa, T., Koide, R., Nakata, Y., Saitsu, H., Matsumoto, N., Takahashi, K. et al. A novel WDR45 mutation in a patient with static encephalopathy of childhood with neurodegeneration in adulthood (SENDA). Am. J. Med. Genet. A 164A, 2388-2390 (2014).

30 Hamdan, F. F., Srour, M., Capo-Chichi, J. M., Daoud, H., Nassif, C., Patry, L. et al. De novo mutations in moderate or severe intellectual disability. PLoS Genet. 10 e1004772 (2014)

31 Hayflick, S. J., Kruer, M. C., Gregory, A., Haack, T. B., Kurian, M. A., Houlden, H. H. et al. Beta-propeller protein-associated neurodegeneration: a new X-linked dominant disorder with brain iron accumulation. Brain 136, 1708-1717 (2013).

32 Kruer, M. C., Boddaert, N., Schneider, S. A., Houlden, H., Bhatia, K. P., Gregory, A. et al. Neuroimaging features of neurodegeneration with brain iron accumulation. AJNR Am. J. Neuroradiol. 33, 407-414 (2012).

33 Gregory, A., Polster, B. J. \& Hayflick, S. J. Clinical and genetic delineation of neurodegeneration with brain iron accumulation. J. Med. Genet 46, 73-80 (2009).

34 Schneider, S. A. \& Bhatia, K. P. Syndromes of neurodegeneration with brain iron accumulation. Semin. Pediatr. Neurol. 19, 57-66 (2012).

35 Gregory, A. \& Hayflick, S. J. Genetics of neurodegeneration with brain iron accumulation. Curr. Neurol. Neurosci. Rep. 11, 254-261 (2011).

36 Ichinose, Y., Miwa, M., Onohara, A., Obi, K., Shindo, K., Saitsu, H. et al. Characteristic MRI findings in beta-propeller protein-associated neurodegeneration (BPAN). Neurol. Clin. Pract. 4, 175-177 (2014).

37 Nakashima, M., Miyajima, M., Sugano, H., limura, Y., Kato, M., Tsurusaki, Y. et al. The somatic GNAQ mutation c.548G $>$ A (p.R183Q) is consistently found in Sturge-Weber syndrome. J. Hum. Genet. 59, 691-693 (2014).

38 Robinson, J. T., Thorvaldsdottir, H., Winckler, W., Guttman, M., Lander, E. S., Getz, G. et al. Integrative genomics viewer. Nat. Biotechnol. 29, 24-26 (2011).

39 Thorvaldsdottir, H., Robinson, J. T. \& Mesirov, J. P. Integrative Genomics Viewer (IGV) high-performance genomics data visualization and exploration. Brief Bioinform. 14, 178-192 (2013).

40 Acuna-Hidalgo, R., Bo, T., Kwint, M. P., van de Vorst, M., Pinelli, M., Veltman, J. A et al. Post-zygotic point mutations are an underrecognized source of de novo genomic variation. Am J. Hum. Genet. 97, 67-74 (2015).

41 Campbell, I. M., Yuan, B., Robberecht, C., Pfundt, R., Szafranski, P., McEntagart, M. E. et al. Parental somatic mosaicism is underrecognized and influences recurrence risk of genomic disorders. Am J. Hum. Genet. 95, 173-182 (2014).

42 Campbell, I. M., Stewart, J. R., James, R. A., Lupski, J. R., Stankiewicz, P., Olofsson, P. et al. Parent of origin, mosaicism, and recurrence risk: probabilistic modeling explains the broken symmetry of transmission genetics. Am J. Hum. Genet. 95, 345-359 (2014). 